\title{
Concordância e tradução para o português do Teste de Habilidade Motora do Membro Superior - THMMS
}

\section{Reliability and Translation to Portuguese of the Arm Motor Ability Test - AMAT}

\author{
Ana Cristina Guedes Morlin ${ }^{1}$, Ana Márcia Delattre ${ }^{1}$, Enio Walker Azevedo Cacho², Telma Dagmar \\ Oberg $^{3}$, Roberta de Oliveira ${ }^{4}$
}

\section{RESUMO}

Objetivo: O presente estudo tem o objetivo de avaliar a concordância e traduzir para a língua portuguesa o Teste de Habilidade Motora do Membro Superior (THMMS). Método: A tradução foi realizada com base na versão original em inglês - Arm Motor Ability Test (AMAT). Este processo contou com um tradutor e pessoal da área da saúde bilíngüe. Quatro fisioterapeutas participaram de um treinamento teórico-prático para a aplicação da escala. Foram avaliados 10 pacientes com Acidente Vascular Encefálico crônico, através do THMMS, do Mini-Exame do Estado Mental e da Secção do Membro Superior da Escala de Desempenho Físico de Fugl-Meyer (FM). A análise dos dados foi realizada através do coeficiente W de Kendall para a concordância entre os examinadores e o coeficiente de Spearman para avaliar a correlação entre as pontuações da THMMS e a FM, utilizando o nível de significância estatístico de $p<0,05$. Resultados: Na tradução para o português do THMMS foi encontrada significativa concordância interobservador para as treze tarefas $(p<0.001)$ e boa relação entre a THMMS e a FM ( $r s=0,8936 ; p<0.05)$. Conclusão: O THMMS é devidamente consistente na avaliação da função do membro superior de indivíduos hemiplégico, facilitando a compreensão e tratamento individualizado.

\section{Unitermos: Reprodutibilidade de Resultados, Acidente cerebrovascular, Reabilitação.}

Citação. Morlin ACG, Delattre AM, Cacho EWA, Oberg TS, Oliveira R. Concordância e tradução para o português do Teste de Habilidade Motora do Membro Superior - THMMS. Rev Neurocienc 2006; 14(2):006-009.

\section{SUMMARY}

Objective: This study has aimed to evaluate the reliability and translation to Portuguese of the Arm Motor Ability Test (AMAT). Method: A bilingual translator of health sciences did the translation based in the American original version and four physiotherapists participated of a theoretical and practice trainee for the application of the scale. AMAT, Mini-Mental Exam and the Fugl-Meyer upper-limb section evaluated ten chronic stroke patients. The date was analyzed through W of Kendall coefficient for the inter-rater reliability and the Spearman's coefficient for the correlation between AMAT and Fugl-Meyer (significant statistical level of $p<0.05$ ). Results: Translating AMAT into Portuguese we found inter-rater reliability for the thirteen tasks $(p<0.001)$ and good relationship between the AMAT and the Fugl-Meyer upper-limb section ( $r s=0.8936 ; p<0.05)$. Conclusion: AMAT is a consistent method for assessment of the hemiparetic upper limb helping the comprehension and the individual treatment.

\section{Keywords: Reliability, Stroke, Rehabilitation.}

Citation. Morlin ACG, Delattre AM, Cacho EWA, Oberg TS, Oliveira R. Reliability and Translation to Portuguese of the Arm Motor Ability Test - AMAT. Rev Neurocienc 2006; 14(2):006-009.

\section{INTRODUÇÃO}

A hemiplegia após uma lesão do Sistema Nervoso Central (SNC) é uma das formas mais prevalentes de danos motores, afetando aproximadamente 1\% da população ${ }^{1}$. A função normal da extremidade superior inclui a capacidade de alcance direcionado, preensão e manipulação de objetos é a base das capacidades motoras requeridas para a realização das Atividades de
Vida Diária (AVD's)².Após uma lesão unilateral do SNC, o paciente pode não ser capaz de usar a extremidade superior envolvida quando a oposta, menos afetada, está disponível ${ }^{3}$. Mesmo em indivíduos com leve hemiparesia, os movimentos de alcance e preensão no membro superior comprometido apresentam lentidão e redução da amplitude de movimento, sendo a trajetória caracterizada por movimentos segmentados, baixa

Trabalho realizado na Universidade Estadual de Campinas - UNICAMP

1 - Fisioterapeuta, Especialista em Fisioterapia aplicada a Neurologia Adulto - FCM/Unicamp

2 - Fisioterapeuta, Doutorando em Cirurgia - FCM/Unicamp

3 - Fisioterapeuta, Doutora em Ciências Médicas - Neurologia FCM/Unicamp

4 - Fisioterapeuta, Mestranda em Ciências Médicas - Neurologia FCM/Unicamp

Endereço para correspondência: Enio Walker A. Cacho - Rua Vitorino Ferrari, 27A, Bairro Barão Geraldo, CEP: 13084-040. E-mail: enio@fcm.unicamp.br 
variabilidade e coordenação interarticular interrompida quando comparados aos indivíduos são4 .

Quando se propõem programas terapêuticos para estes comprometimentos, a identificação dos problemas específicos, bem como a quantificação e estabelecimento de prioridades, exige a documentação da funcionalidade de forma compreensível e reprodutível. Um componente essencial da avaliação da efetividade de um programa de reabilitação é a coleta sistematizada de dados com o acompanhamento do desempenho funcional dos pacientes ${ }^{5}$.

São vários os testes que se direcionam para a avaliação da habilidade do membro superior hemiparético durante as AVD's, todos apresentando vantagens e desvantagens. Entretanto, poucos são utilizados no nosso meio. Na busca por medidas objetivas que forneçam uma base científica para comunicação entre profissionais, documentação da eficácia de um tratamento e credibilidade dentro da pesquisa em saúde, encontramos alguns, dentre eles: o Teste do Braço de Frenchay ${ }^{6}$, o Teste da Função da Mão de Jebsen-Taylor ${ }^{7}$, o Teste de Função Motora de Wolf8, TEMPA ${ }^{9}$ e o Teste de Habilidade Motora do Membro Superior - $\mathrm{THMMS}^{10}$. No entanto, nenhum se encontra traduzido para o português e com testes de concordância realizados no Brasil.

O THMMS foi desenvolvido em 1988 para mensurar aspectos quantitativos e qualitativos das AVD's envolvendo o membro superior de pacientes com Acidente Vascular Encefálico (AVE) ${ }^{11}$. Em 1997, Kopp et al ${ }^{10}$ verificou sua confiabilidade, validade e sensibilidade como um instrumento de avaliação das inabilidades nas AVD's envolvendo o membro superior. Os testes também classificam cada componente da tarefa de acordo com duas escalas importantes para recuperação da função motora: Habilidade Funcional (capacidade de executar uma meta) e Qualidade de Movimento (quão bem o movimento da tarefa foi executado), fornecendo informações sobre os aspectos do movimento que são difíceis de verificar quantitativamente quando estudados em uma ampla série de tarefas.

Diante do exposto acima, este trabalho tem o objetivo de avaliar a concordância e traduzir para a língua portuguesa o Teste de Habilidade Motora do Membro Superior - THMMS.

\section{MATERIAL E MÉTODO}

O estudo contou com 10 pacientes portadores de seqüela de AVE crônico, seis homens e quatro mulheres entre 17 e 63 anos de idade (média 44,8 \pm 12,9), com tempo de lesão variando de 1 a 10 anos, sendo que sete apresentavam hemiparesia à direita e três hemiparesia à esquerda. Foram excluídos portadores de déficits cognitivos severos.

\section{Instrumentos de Medidas}

O Teste de Habilidade Motora do Membro Superior (THMMS) é composto por 13 tarefas que reproduzem atividades cotidianas, avaliadas por uma escala que varia de 0 a 5 nos itens: (a) habilidade funcional - onde $0=$ não tem, 1 = muito pouco, 2 = pouco, 3 = modera- do, 4 = quase normal e 5 = normal; e (b) qualidade do movimento - 0 = não tem, $1=$ muito pobre, $2=$ pobre, 3 $=$ moderado, $4=$ quase normal e $5=$ normal. $O$ tempo de desempenho de cada tarefa é cronometrado e pode variar de 60 a 120 segundos (Anexo 1) ${ }^{10}$.

Anexo 1. Teste de Habilidade Motora do Membro Superior
1.Cortar "carne"

Pegar garfo e faca

Cortar carne ${ }^{a, b}$

Garfo até a boca

Pegar sanduíche Levar até a boca

3. Comer com colher Pegar colher

Pegar sopa com a colher

Colher até a boca

4. Beber em caneca

Segurar no cabo da caneca

Caneca até a boca

5. Pentear o cabelo

Pegar o pente

Pentear o cabelo ${ }^{b}$

6. Abrir a jarra

Segurar a tampa da jarra ${ }^{a}$

Apertar e abrir a jarra ${ }^{a}$

7. Dar nó em cadarço

Dar nó em cadarço ${ }^{\mathrm{a}, \mathrm{b}}$
2. Comer sanduíche

\section{Usar o telefone \\ Levar o receptor do telefone à orelha \\ Digitar um número de telefone \\ 9. Secar água derramada Secar a água em até seis mo- vimentos \\ Torcer o pano}

10. Colocar o casaco

Braço afetado na manga, colocar o casaco, pelo ombro afetado ${ }^{a, b}$ Abotoar dois botões ${ }^{a}$

\section{Colocar a camiseta}

Braços nas mangas da camiseta ${ }^{a, b}$ Cabeça pela gola $a, b$

Colocar e ajeitar a camiseta ${ }^{a}$

12. Estender o braço

Estender o braço afetado e pegar um objeto pequeno ${ }^{a}$

13. Acender a luz/abrir a porta Acender a luz pelo interruptor Agarrar a maçaneta, rodar, abrir a porta em até seis movimentos Fechar a porta
${ }^{a}$ Atividades bilaterais; ${ }^{b}$ tempo limite de 2 minutos (demais tarefas têm tempo limite de 1 minuto) Fonte: Adaptado de Kopp B, Kunkel A, Flor H, et al. The Arm Motor Ability Test: Reliability, Validity and Sensibility to Change of an Instrumentfor Assessing Disabilities in Activities of Daily Living. Arch Phys Med Rehab 1997; 78: 615-20.

O Mini-Exame do Estado Mental (MEEM) ${ }^{12}$ é utilizado para detectar problemas cognitivos. Envolve duas categorias de respostas verbais e não verbais. Os sub-testes verbais medem, em particular, orientação espaço-temporal, memória imediata, memória de evocação, atenção e linguagem. Os sub-testes não verbais medem a coordenação perceptiva motora e a compreensão de instruções. O MEEM é composto por trinta questões categóricas e a pontuação é feita da seguinte forma: de 30 a 26 pontos a função cognitiva está preservada, de 26 a 24 pontos há alteração não sugestiva de déficits e 23 ou menos pontos é sugestivo de déficits cognitivos.

A Secção do Membro Superior da Escala de Desempenho Físico de Fugl-Meyer (FM) é uma compreensiva mensuração do comprometimento motor após uma lesão, relatando padrões sinérgico e coordenação do ombro, antebraço, punho e mão ${ }^{13}$. Os resultados são obtidos em uma escala ordinal na qual $0=$ não realiza, 1 = consegue parcialmente e 2 = consegue completamente, resultando no score máximo de 66 pontos para o membro superior (Anexo 2).

\section{Procedimentos}

\section{1 .Tradução}

Para que a aplicação do THMMS fosse possível, foi realizada sua tradução com base em sua versão original em inglês (Arm Motor Ability Test - AMAT) ${ }^{10}$. 
Anexo 2. Secção de Membro Superior da Fugl-Meyer

1. Ombro, cotovelo e antebraço (máximo 36 pontos)

- Presença de reflexos anormais: bíceps, tríceps e flexores dos dedos.

- Movimentos voluntários na sinergia

\section{Sinergia flexora}

Retração do ombro, elevação, abdução, rotação externa.

Flexão do cotovelo

Supinação do antebraço

\section{Sinergia extensora}

Abdução e rotação interna do ombro

Extensão do cotovelo

Pronação do antebraço

- Combinação de movimentos voluntários dinâmicos na sinergia flexora e extensora

Mão coluna lombar

Flexão do ombro de 0 até 90 graus com extensão do cotovelo

Prono-supinação do antebraço com flexão do cotovelo

- Presença de reflexos normais: bíceps, tríceps e flexores dos dedos.
2. Punho (máximo de 10 pontos)

- Extensão estática do punho em $15^{\circ} \mathrm{com}$ cotovelo fletido

- Flexão e extensão repetida do punho com cotovelo fletido

- Extensão estática do punho para $15^{\circ} \mathrm{com}$ o cotovelo estendido

- Flexão e extensão repetida do punho com o cotovelo estendido

- Circundução

3. Mão (máximo de 14 pontos)

- Flexão em massa dos dedos

- Extensão em massa dos dedos

- Preensão em gancho

- Preensão radial

- Preensão em pinça

- Preensão de um cilindro

- Preensão esférica

4. Coordenação e velocidade durante o teste index - nariz (máximo de 6 pontos)

- Presença de tremor

- Presença de dismetria

- Avaliação da velocidade

Fonte: Adaptado de Cacho EWA, Melo FRLV, Oliveira R. Avaliação da Recuperação motora de pacientes hemiplégicos através do protocolo de desempenho físico de Fugl-Meyer. Rev Neurociências 2004; 12 (2): 94-102.

Este processo contou com um tradutor e pessoal da área da saúde bilíngüe familiarizado com a natureza do estudo, enfatizando-se a tradução semântica e cultural e não a estritamente literária. Foi realizada a versão do instrumento em português novamente ao inglês, mas não foram encontrados conflitos de interpretação.

Visto que a aplicação deste instrumento não se faz por meio de auto-aplicação, mas por intermédio dos avaliadores, foi realizada uma avaliação-teste entre os profissionais que iriam posteriormente utilizar-se deste instrumento para avaliar os pacientes. Nesta avaliação-teste questionou-se a relevância de cada um dos itens abordados pela THMMS no contexto cultural brasileiro, além da dificuldade de entendimento e realização dos itens assim como a necessidade de inclusão ou exclusão de algum assunto em particular.

\section{Treinamento dos avaliadores}

Um conjunto de quatro profissionais da saúde - fisioterapeutas experientes na área - participaram de um treinamento teórico-prático, no qual foram expostos os critérios que seriam utilizados para as pontuações de cada item e esclarecimento sobre a avaliação com o intuito de promover uma padronização na habilidade do uso do instrumento. Os pacientes que participaram do treinamento prático não foram selecionados para a amostra deste estudo.

\section{Avaliação}

Os pacientes selecionados realizam acompanhamento no Ambulatório de Fisioterapia e Terapia Ocupacional do Hospital de Clínicas da Unicamp (HC/UNICAMP). Os indivíduos foram avaliados por um examinador nos testes do MEEM e a Secção de Membro Superior de FM. Em seguida os pacientes eram encaminhados a uma sala onde estavam os quatros examinadores, onde era aplicado o THMMS.

O paciente recebia do examinador instruções e duas demonstrações de cada tarefa utilizando o mesmo braço que o paciente deveria usar. Nas tarefas bimanuais era enfatizado a utilização do membro parético. Nas tarefas unimanuais não era permitido a prática com o membro são.

As avaliações eram feitas simultaneamente pelos quatro avaliadores que observavam atentamente o desempenho dos pacientes executando a tarefa solicitada. As notas eram dadas conforme a qualidade do movimento, isto é, na precisão que era realizado e na habilidade funcional (pontuava se ao final do movimento o paciente havia executado ou não a tarefa solicitada). Em todas as tarefas, sem a percepção do paciente - para não afetar o desempenho - o tempo era cronometrado para que não ultrapassasse o limite de 60 ou 120 segundos. Se fosse ultrapassado, o paciente era instruído a encerrar a tarefa. Neste caso, a habilidade funcional era nula, mas a qualidade de movimento era analisada até a interrupção da execução.

\section{Análise dos Dados}

Para a análise de concordância entre os examinadores foi utilizado o coeficiente W de Kendall e para avaliar a correlação entre as pontuações numéricas da THMMS e a Secção de Membro Superior de FM foi utilizado o coeficiente de correlação de Spearman. O nível de significância adotado no testes estatístico foi de $p<0.05$.

\section{RESULTADOS}

Foi encontrada significativa concordância interobservador para as treze tarefas da THMMS com $p<0.001$ (Tabela 1). Fora encontrado também uma boa relação entre a THMMS e a Secção de Membro Superior FM (rs $=0,8936 ; p<0.05)$.

\section{DISCUSSÃO}

Uma avaliação eficaz, para relatar com clareza e precisão o grau de inabilidade motora do paciente com injúria do SNC é imprescindível no meio científico. No 
entanto, a maioria dos instrumentos é quase que exclusivamente encontrados em língua estrangeira.

Tabela 1. Análise de concordância entre os examinadores para cada atividade do Teste de Habilidade Motora do Membro Superior - THMMS.

\begin{tabular}{lc}
\hline \multicolumn{1}{c}{ Atividades } & W de Kendall \\
\hline 1 - Cortar carne & 0,974 \\
2 - Comer sanduíche & 0,947 \\
3 - Comer com colher & 0,966 \\
4 - Beber em caneca & 0,946 \\
5 - Pentear o cabelo & 0,933 \\
6 - Abrir a jarra & 0,898 \\
7 - Amarrar o cadarço & 0,925 \\
8 - Usar o telefone & 0,932 \\
- Secar água derramada & 0,948 \\
10 - Colocar o casaco & 0,963 \\
11 - Colocar camiseta & 0,908 \\
12 - Estender o braço & 0,879 \\
13 - Acender a luz/abrir a porta & 0,878 \\
\hline 0,001 &
\end{tabular}

Uma das finalidades primárias deste estudo não foi somente traduzir este instrumento, mas trazê-lo para um contexto cultural específico, respeitando aspectos primordiais como a equivalência experimental ou cultural vivida pela população a qual se destina. Para sua reprodução na língua portuguesa preservamos todas as tarefas que foram realizadas na versão original em inglês e através de questionamentos entre os avaliadores, deparamo-nos com algumas dificuldades. Dentre elas, a atividade 'dar nó em gravata' (Item VII - Anexo 1) foi a mais trabalhosa, não exclusivamente pelo acometimento motor, mas pela falta de adequação sócio-econômica e cultural de nossa população. Como sugestão para a tarefa - preservando o grau de dificuldade - utilizamos 'dar nó em cadarço', trazendo-a mais próxima de nossas atividades cotidianas. Outro empecilho encontrado foi nas tarefas referentes à alimentação - cortar carne, comer sanduíche e tomar sopa, pela dificuldade de se encontrar tais aparatos em ambiente ambulatorial e/ ou hospitalar.

Mesmo que criada exclusivamente para determinar a eficácia da terapia de restrição induzida, cuja essência era reduzir a probabilidade de uso do membro não afetado, o THMMS adaptou-se perfeitamente como um método de avaliação independente do tratamento realizado.

A THMMS abrange várias AVD's que incluem atividades bimanuais e priorizam o membro comprometido. Isto favorece a descrição do padrão de recuperação funcional, diminuído a influência compensatória da extremidade não-afetada na função motora final.

Como encontrando em nosso estudo, Chae $(2003)^{14}$ também relata uma boa relação entre a THMMS e a Secção de Membro Superior de FM, mesmo observando que a primeira avalia a capacidade e habilidade funcional e a segunda, o nível de comprometimento motor. Foi relatada pelos examinadores que houve uma maior dificuldade em pontuar os pacientes com a THMMS quando os mesmos apresentavam comprometimento moderado na secção de membro superior da FM.

Um outro ponto a ser levantado é o tempo gasto com a aplicação da THMMS. Enquanto a execução da secção de membro superior da FM levou em média um período de 10 minutos, o THMMS consumiu de 30 a 40 minutos, produzindo desgaste em alguns pacientes.

\section{CONCLUSÃO}

O THMMS é devidamente consistente e eficaz para avaliação da função do membro superior durante as AVD's, pois reproduz no ambiente ambulatorial e/ou hospitalar situações muito próximas às encontradas no nosso cotidiano. Com isto, os profissionais ligados à reabilitação podem claramente avaliar e ampliar seus conhecimentos sobre o estado exato da função do membro superior do paciente hemiplégico, para que assim, possa traçar um tratamento eficiente e individualizado.

\section{AGRADECIMENTOS}

Aos fisioterapeutas e colaboradores Leonardo de Pereira Binda, Ângela Salomão Macedo e Renata Vieira de Moraes.

\section{REFERÊNCIAS BIBLIOGRÁFICAS}

1. Penta M, Luigi T. The ABILHAND Questionnaire as a Measure of Manua Ability in Chronic Stoke Patients. Stroke 2001: 32(7):1627-1634.

2. Shumway-Cook A, Woollacott MH. Controle Motor - Teorias e aplicações práticas. 2a ed. Barueri: Manole; 2003.

3. Van der Lee $\mathrm{JH}$. Constraint-induced therapy for stroke: more of the same or something completely different? Curr Opinion Neurol 2001; 14: 741-744.

4. Zackoski KM, Dromerick AW, Sahrmann SA, Thach WT, Bastian AJ. How do strength, sensation, spasticty and joint individuation relate to the reaching deficits of people with chronic hemiparesis? Brain 2004; 127: 1035-1046.

5. Bear GD, Smith MT, Rowe PJ, Masterton L. Standardized physical therapy measurements for assessing impairment and disability following stroke. Phys Med Rehab 2003; 84: 977-981.

6. Parker VM, Wade DT, Langton Hewer R. Loss of arm function after stroke: measurement, frequency and recovery. Rehab Med 1986; 8: 69-73.

7. Jebsen RH, Taylor N, Trieshmann RB, Trotter MJ, Howard LA. An objective and standardized test of hand function. Arch Phys Med Rehab 1969; 50: 311-319.

8. Wolf SL, Lecraw DE, Barton LA, Jann BB. Forced use of hemiplegic upper extremities to reverse the effect of learned nonuse among chronic stroke and head-injured patients. Exp Neurol 1989; 104: 125-132.

9. Desrosiers J, Hérbert R, Dutil E, Bravo G. Development and reliability of an upper extremity function test for the elderly: the TEMPA. Can J Occup Ther 1993; 60: 9-16.

10. Kopp B, Kunkel A, Flor H, Platz T, Rose U, Dipl-Psych, et al. The Arm Motor Ability Test: Reliability, Validity and Sensibility to Change of an Instrument for Assessing Disabilities in Activities of Daily Living. Arch Phys Med Rehab 1997: 78: 615-620.

11. McCulloch K, Cook EW III, Fleming WC, Novack TA, Taub E. A Reliable Test of Upper Extremity ADL Function. Arch Phys Med Rehab 1988; 69: 755.

12. Folstein S, Folstein M, McHugh PR. "Mini-Mental State". A practical method for grading the cognitive state of patients for the clinician. J Phsychiat 1975; 12: 189-198.

13. Cacho EWA, Melo FRLV, Oliveira R. Avaliação da Recuperação motora de pacientes hemiplégicos através do protocolo de desempenho físico de FuglMeyer. Rev Neurociências 2004; 12 (2): 94-102. Errata 2004; 12 (4): 221.

14. Chae J. Labatial, Yang G. Upper limb motor function in hemiparesis: Concurrent validity of the arm motor ability test. Am J Phys Med Rehabil 2003; 82: 1-8. 